\title{
Performance Evaluation of Inversion Mode and Junctionless Dual-Material Double-Surrounding Gate Si Nanotube MOSFET for 5-nm Gate Length
}

\section{(C) Sanjay, B. Prasad, A. Vohra}

Electronic Science Department, Kurukshetra University,

Kurukshetra-136119, Haryana, India

E-mail: sanjaykumarvlsi2015@gmail.com

Received October 27, 2020

Revised December 7, 2020

Accepted for publication December 9, 2020

In this work, drain current $I_{D}$ for 5-nm gate length with dual-material (DM) double-surrounding gate (DSG) inversion mode (IM) and junctionless (JL) silicon nanotube (SiNT) MOSFET have been studied and simulation results are reported using Silvaco ATLAS 3D TCAD. For this work, we used the non-equilibrium Green's function (NEGF) approach and self-consistent solution of Poisson's equation with Schrödinger's equation. The conduction band splitting into multiple sub-bands has been considered and there is no doping in channel in case of IM SiNT MOSFET. The effect of DM gate engineering for SiNT channel radius $1.5 \mathrm{~nm}$ with $0.8-\mathrm{nm}$ gate oxide $\left(\mathrm{SiO}_{2}\right)$ thickness on $I_{D}$ has been studied. A comparison of results has been done between IM DM DSG and JL DM DSG CGAA SiNT. In case of JL, doping concentration is optimized for two concerns: (i) to get the same $I_{\text {On }}$ current as IM device and (ii) to get the same threshold voltage $V_{\mathrm{Th}}$ as IM. This has resulted in $10^{2}$ and $10^{3}$ times smaller $I_{\text {Off }}$ in matching $I_{\mathrm{On}}$ and $V_{\mathrm{Th}}$ optimized device, respectively, as compared to IM. It is found that DM gate engineering reduces drain-induced barrier lowering (DIBL) for both IM and JL SiNT MOSFET. In this work, JL have much smaller DIBL $\sim 15 \mathrm{mV} / \mathrm{V}$, almost an ideal $\mathrm{SS} \sim 60 \mathrm{mV} / \mathrm{dec}$, and higher $I_{\mathrm{On}} / I_{\mathrm{Off}}$ ratio $\sim 2.18 \cdot 10^{8}$ as compared to available CGAA literature results.

Keywords: inversion mode, junctionless, DM DSG, Si nanotube MOSFET, NEGF, $I_{D}$, SS, DIBL.

Full text of the paper will appear in journal SEMICONDUCTORS. 